\title{
The control of saccade size and fixation duration in reading: The limits of linguistic control
}

\author{
J. KEVIN O'REGAN \\ Centre National de la Recherche Scientifique, Paris, France
}

\begin{abstract}
This experiment investigates how information about six letter spaces to the right of the current fixation point is used to guide the eye in reading. It is found that low-quality cues such as word length can be extracted from this region sufficiently quickly to influence the size of the immediately following saccade. Linguistic processing of information from this region is also done, but only begins to influence the eye's behavior at the next fixation point, where fixation duration is affected. Subsequent eye-movement characteristics are more strongly influenced, but this influence is diffuse, that is, spread over a variety of eye-movement parameters, and takes about 1 sec to develop.
\end{abstract}

Several studies have suggested that the saccades made by the eye during reading vary in size from moment to moment as a function of textual parameters such as word length (O'Regan, 1975; Rayner \& McConkie, 1976), spacing (Abrams \& Zuber, 1972; McConkie \& Rayner, 1975), and identity of words (O'Regan, 1979). As noted in O'Regan (1975), two models of eye-movement control are compatible with such variations in saccade size. According to the "length control" model, the eye adjusts its saccades purely on the basis of information concerning the length of the words in the nearby visual field. For example, a strategy such as "jump to the middle of the nearest word in noncentral vision"' would be compatible with much of the data. Alternately, under the "linguistic control" model, it is supposed that both purely visual information about the identity of letters, and linguistic information (in particular, information about words in the lexicon) are combined and used to constrain the number of choices that have to be made in identifying words. At each fixation, this process of combining visual and linguistic information defines a region of good "perceptibility": The next saccade is programmed to lead the eye just beyond this region. "Perceptibility" was distinguished from "visibility" in O'Regan (1979). The "visibility" of a given letter in a given position of a line is the probability of reporting that letter without making use of linguistic constraints (that is, as if it were

I warmly thank Virginia Holmes for her encouragement during the experimental phase of this research, and John Findlay and Ariane Lévy-Schoen for their encouragement during the writing phase. I also thank K. Rayner and a reviewer for helpful comments. Please send reprint requests to me at: Laboratoire de Psychologie, 54 Bd Raspail, Paris 6, France. inserted in a random letter sequence). "Perceptibility" is the probability of reporting that letter when, in addition, all possible lexical, syntactic, and semantic constraints are taken into account.

Hypotheses similar to the linguistic control hypothesis have been proposed by Haber (1976), Hochberg (1970), and Rayner and McConkie (1976). O'Regan (1979) reported two experiments that supported the linguistic control hypothesis by showing that the identity of words to the right of the current fixation point influenced saccade sizes immediately. In one experiment, it was shown that, having scanned a series of words, the eye then jumped further if the next word was THE than if it was a three-letter verb. The difference in saccade sizes was attributed to the fact that the three-letter verbs were less common than THE, it being hypothesized that they were therefore harder to decode from the visual cues. More weight was given to this argument through the finding that the differences found were smaller when the relatively common auxiliaries HAD, WAS, and ARE were compared with THE than when the rarer verbs ATE, MET, and RAN were compared with THE. In this experiment, the effect of linguistic control was only active very close (three letters) to the momentary fixation point.

Another experiment by O'Regan (1979) tried to show linguistic control acting as a function of information farther than seven letter spaces from the momentary fixation point. Subjects reading sentences under conditions of normal visibility made longer saccades approaching or leaving long words than approaching or leaving short words. This is expected under linguistic control, since the zone of perceptibility generally extends to the end of the nearest word, be it 
long or short, because the strongest constraints, generally lexical constraints, act only within words. However, the same behavior is also predictable by a length-control strategy.

In the reduced peripheral vision condition, subjects read sentences through a moving "window": At every instant, all letters beyond seven character spaces from the momentary fixation point were replaced by $\mathrm{Xs}$ so that information about individual letters was destroyed but information about word length was retained (McConkie \& Rayner, 1975, used similar window conditions). If saccades are controlled by linguistic information, then the eye should now no longer behave differently when approaching long, as compared with short, words. But if saccades are controlled only by length information, the eye should still behave differently when approaching long, rather than short, words. The difference in saccade size approaching long, as opposed to short, words still existed, but was significantly smaller in the reduced peripheral vision condition. The result argues for the existence of some degree of linguistic control as a function of information gathered beyond seven letters from the momentary fixation point. However, this result must be treated with caution, because the group of subjects who read in the reduced peripheral vision condition had Xs in periphery all the time and may therefore have adopted a different reading strategy from the normal group, even though they reported being completely unaware of the presence of the Xs.

The present experiment addressed this problem by having the same group of subjects read sentences occurring under both reduced and normal peripheral vision. To further diminish any perturbation caused by reducing peripheral vision, only a small "critical region" in each test sentence experienced a display change, and many nonchanging filler sentences were used.

\section{METHOD}

\footnotetext{
Materials and Subjects

Thirty-two sentence frames were constructed. Each sentence frame contained a "critical region," 9-14 letters in length (mean, 12 letters), which could be filled in four ways, corresponding to four experimental conditions, as shown in Table 1. The first word in the critical region could be long or short; the content of peripheral vision before the critical region was fixated could be normal or inappropriate. In the "normal-short" (N-S) condition, the critical region was filled with a three-letter word followed by a medium-length word. In the "inappropriate-short" (I-S) condition, the critical region contained the same three-letter, medium-length word combination. However, this was displayed by the computer only after the eye passed an imaginary boundary six character spaces to the left of the critical region. Before this, that is, while the critical region was still in peripheral vision, the two words were displayed written backward, thereby destroying the lexical information usually available in peripheral vision from the critical region (by making letter information inappropriate), but retaining wordlength information. Display changes look place while the eye was making a saccade, defined as a movement faster than one letter in
}

Table 1

Examples of Contents of a Critical Region for Four Conditions

\begin{tabular}{ccc}
\hline & $\mathrm{N}$ & \multicolumn{1}{c}{ I } \\
\hline \multirow{2}{*}{$\mathrm{S}$} & CRI ÉTOUFFÉ & IRC ÉFFUOTÉ \\
& & CRI ÉTOUFFÉ \\
& & TNEMESSIMÉG \\
L & GÉMISSEMENT & $\downarrow$ \\
& & GÉMISSEMENT \\
\hline
\end{tabular}

Note-This particular critical region was preceded by "Les voisins soutenaient que le..." and followed by "... venait de la cave de la maison." The arrow indicates that the first sequence of letters was transformed into the second one when the subject's eye passed the imaginary boundary (/) in "... soutenaient qu/ele...."

10 msec. The "critical region" and "imaginary boundary" techniques were first used by Rayner in 1975 .

In the "normal-long" ( $\mathrm{N}-\mathrm{L})$ condition, the critical region was filled with one long word. In the "inappropriate-long" (I-L) condition, the long word was displayed backward until the eye first came within six character spaces to its left, and then the long word was displayed normally.

The present method of reducing the content of peripheral vision was used because pilot experiments had shown that if Xs were used, as in O'Regan (1979), subjects were aware of a repetitive pattern to the right of their fixation point. However, if random letters (approximated by writing the word backwards) were used, the subjects were unaware of anything abnormal. Two differences with the O'Regan (1979) experiment may explain why, in that experiment, subjects did not see the Xs in periphery. First, letters were of a different typeface. Second, reduced peripheral vision subjects always had Xs in peripheral vision, and this may have globally reduced their attention span. As will be seen later, this hypothesis is favored by the results of the present experiment.

The 32 sentence frames were divided into four sets of eight. A Latin square design was used to assign these four sentence-frame sets to the four critical zone types in four different ways for each of four groups of subjects. The 24 subjects were native French speakers, six in each of the four groups. The 32 test sentences were pseudorandomly intermixed with 54 filler sentences. The experiment began with 10 practice sentences. Each of the 96 sentences read by the subjects was followed by a question, to which the subjects responded "yes" or "no" by pressing a button.

\section{Apparatus}

The sentences were displayed one by one in the middle of a Digital Equipment Corporation VR12 screen, in uppercase letters $(4 \times 6$ matrix of dots). The screen could display 84 characters on a line.

The eye-movement measuring device detected photoelectrically the position of the scleral boundary. The horizontal eye-position signal was amplified by a dc amplifier with a rise time of $.5 \mathrm{msec}$ and was sampled every $10 \mathrm{msec}$ by the analog-to-digital converter of a PDP-12 computer. By the use of an "inverted calibration table" (O'Regan, 1978), always present in the computer memory, each voltage sample was immediately converted to the sentenceposition value, measured in units of one-quarter of a letter, corresponding to the momentary location of the subject's eye. The accuracy to which the computer determined these values depended on the accuracy of the calibration table. The "zero drift" method (O'Regan, 1978) of verifying the calibration table was used after calibration and ensured that, for the viewing distance being used, accuracy was always better than one letter. Use of "letters" rather than degrees as the criterion for calibration accuracy and as the unit of eye position means that the computer can make on-line changes in the display as a function of position in the sentence rather than of visual angle. 
The display was refreshed after each eye-position sample was taken (i.e., every $10 \mathrm{msec}$ ). In the inappropriate peripheral content condition, the word or words in the critical region were at first displayed written backward. After the eye passed an imaginary boundary, located at the left-hand edge of the fifth character space preceding the critical region, the computer continued sampling and displaying the words backwards until the instantaneous velocity of the eye, measured by taking the difference between the current and the preceding eye-position samples, exceeded one letter per $10 \mathrm{msec}$. At that moment, the display was changed so that the word or words in the critical region were correctly displayed. Since it took less than $10 \mathrm{msec}$ to refresh the display, the display change took place less than $10 \mathrm{msec}$ after eye velocity reached the critical value of one letter per $10 \mathrm{msec}$. The shortest duration saccade that can attain this speed (about $25-50 \mathrm{deg} / \mathrm{set}$ ) is of $25 \mathrm{msec}$ duration, and the speed is attained at its halfway point. Even in the worst case, therefore, the display change will take place before the saccade ends.

The distance of the imaginary boundary from the critical region was chosen as six character spaces to ensure that the words written backwards were never visible in central vision. The instant in time when the display change occurred was stored in memory together with the eye-movement record for each sentence. For compatibility with the inappropriate peripheral content condition, the position in the normal condition where the display change would have occurred was calculated and stored in the same way.

\section{Procedure}

Because of the apparatus, subjects could not wear spectacles. They were therefore allowed to choose their own viewing distance from the 20-cm-wide screen, provided it lay between 25 and $50 \mathrm{~cm}$. Subjects unable to read in this range were rejected. Use of the variable viewing distance procedure will, at worst, do no more than introduce noise into the data. In fact, the arguments of Footnote 1 predict that, measured in letter spaces, saccade size should be independent of viewing distance. This was confirmed experimentally by Javal (1879) and Huey (1900). Gilliland (1923) showed a similar adaptation to changes in letter size.

The subject lay back with his head firmly held stationary by the use of a dental imprint. The experimenter adjusted the eyemovement glasses, and the subject performed a "smooth pursuit" calibration, which was verified to an accuracy of one letter by the "zero drift" method (O'Regan, 1978). The experiment then began. A fixation point appeared on the left of the screen and the subject looked at it. If the computer detected a fixation longer than $100 \mathrm{msec}$ within one-half of a letter of the fixation point, the point disappeared and a sentence appeared immediately. If, through calibration error, a difference of more than one-half of a letter between eye position and fixation location occurred, the fixation point remained visible and the computer waited for more accurate fixations. If none occurred after a while, the subject could press a button to recalibrate. Subjects did not usually recalibrate more than once or twice during the experiment.

After a sentence appeared, it remained visible until the subject made a large regression starting from a point beyond the 60th letter of the sentence. When this occurred, the sentence disappeared and a question appeared about $3 \mathrm{~cm}$ above where the sentence had been on the screen. The subject indicated his response by pressing one of two buttons, at which point the left fixation point reappeared and the cycle repeated.

\section{RESULTS}

\section{Analysis of Raw Data}

The number of incorrect responses to test sentences was small (4\%). The subjects were asked, after the experiment, whether they had noticed "anything peculiar about the way in which the letters were dis- played." None of them had. When an example of a display change was shown to them, 2 of the 24 subjects said they "might" have seen such changes.

The eye-movement records, consisting of $10-\mathrm{msec}$ samples of the eye's motion for each sentence, were analyzed to extract the positions and durations of fixations and the size of saccades. A saccade was defined as a movement in which two consecutive $10-\mathrm{msec}$ samples were in the same direction and together covered a distance of more than one letter. A fixation was defined as the interval between the beginnings of successive saccades (this definition counts the duration of the saccade preceding a fixation as being part of the duration of that fixation). The position of a fixation was taken as the last position occupied by the eye before the saccade. Saccade size was defined as the distance between two fixations.

For each record, the first fixation that was made after the display change occurred (or would have occurred) was called fixation 0 . Fixation 0 was always in, or just left of, the critical region. Fixations occurring after fixation 0 were called $1,2,3$, etc., and fixations occurring before fixation 0 were called $-1,-2$, -3 , etc. The saccades were labeled by their starting and ending fixations; for example, the saccade leading from fixation -2 to fixation -1 was called saccade $-2 /-1$. Note that, depending on where fixations lie in the sentence, a saccade can be either a regression or a progression. If fixation -2 lies left of fixation -1 , saccade $-2 /-1$ is a progression; if right of fixation -1 , it is a regression.

\section{Progression Sizes}

For each subject, the mean progression size across the eight sentences read in each condition was calculated for saccade types $-2 /-1,-1 / 0,0 / 1,1 / 2$, and $2 / 3$. These means were subjected to an analysis of variance that took subjects as a random factor (structure: Subjects within Groups by Word Length by Peripheral Content). A separate analysis was done for each saccade type.

The primary purpose of the experiment was to try to confirm that the difference in progression sizes made by the eye approaching a long vs. a short word decreased in the Inappropriate Peripheral Content conditions. This pattern would produce an interaction between Peripheral Content and Word Length for the saccade leading into the critical region, that is, progression $-1 / 0$. The analyses of variance, however, did not reveal this interaction: For the five saccade types, only the main effect of Word Length was significant, and then only for types $-1 / 0,0 / 1$, and $1 / 2$ $[F(1,20)=12.1,16.1$, and 4.4 , respectively, $p<.05$ for each]. See Figure 1. (Note that the absence of any effects at saccades $-2 /-1$ and $2 / 3$ is expected, as these saccades occurred a long way from the critical region.) 


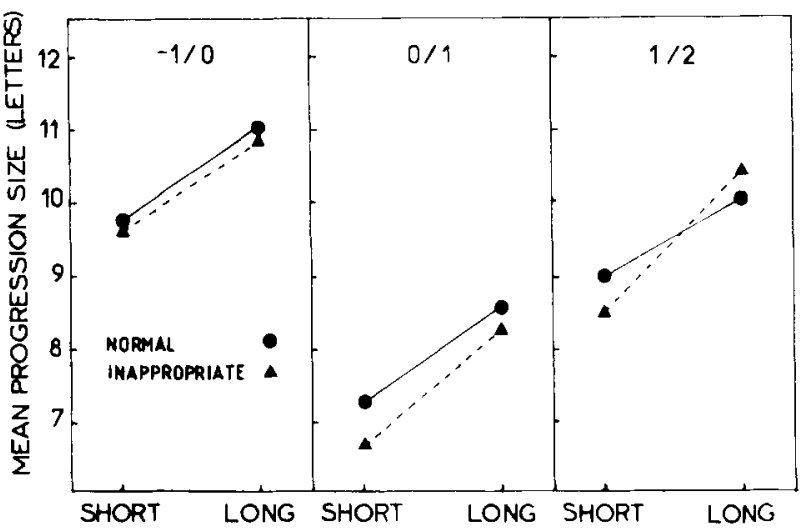

LENGTH OF WOROS IN CRITICAL REGION

Figure 1. Mean progression sizes for saccades $-1 / 0,0 / 1$, and 1/2. SHORT: critical region contains 3-letter word followed by medium-length word. LONG: critical region contains one long word. NORMAL and INAPPROPRIATE refer to the content of the critical region when the eye is at fixation -1 , i.e., when the critical region has not yet been fixated and lies in the right-hand periphery.

The inappropriate peripheral content when the eye was at fixation -1 thus did not produce a decrease of the Word Length effect, either immediately (saccade $-1 / 0$ ) or at a later time (saccades $0 / 1,1 / 2,2 / 3$ ). Therefore, in the conditions of this experiment, information about individual letters beyond six character spaces from the fixation location does not influence saccade size. Rather, the size of the next progression is determined predominantly by wordlength information. The effect of "reduced" peripheral vision found in the window-condition experiment of O'Regan (1979) was therefore probably due to a global perturbation caused by the continual presence of Xs in peripheral vision.

Taking together the present result and the THEskipping result of O'Regan (1979), it is concluded that within six to seven letters of the fixation point, linguistic information and visual information can combine to influence the size of the next progression. But beyond about seven letters, this process breaks down, and word length becomes a controlling factor.

\section{DISCUSSION}

\section{Slower Kinds of Control}

There are two hypotheses consistent with the breakdown of linguistic control of saccades beyond seven letters from the fixation point. It might be that individual letter information beyond seven letters from the fixation point is simply not processed at all. This would be surprising, since such information is certainly available (in particular, as shown by Bouma, 1973, letters flanked by spaces can be seen as far as 20 character spaces from the fixation point). A second possibility is that information is picked up, but, because it is of poorer quality, processing cannot proceed fast enough to influence the immediately following saccade, that is, saccade $-1 / 0$. If this were true, some subsequent aspect of eye-movement behavior might nevertheless be influenced, in particular, fixation duration 0 . This would be evidence for a "slower" version of the linguistic control hypothesis.

Analyses of variance were done on fixation durations at fixations $-2,-1,0,1$, and 2 for the four experimental conditions. Statistics and structure were as before for progression sizes: Subjects within Groups by Word Length by Peripheral Content. There were significant effects only for fixations 0 and 1 (see Figure 2). At fixation 0 , both Word Length $[F(1,20)=$ $4.3, p<.05]$ and Peripheral Content $[F(1,20)=6.6$, $p<.05]$ were significant, although their interaction was not $[F(1,20)=2.3, p>.1]$. At fixation 1 , only Word Length was significant $[F(1,20)=4.4, p<.05]$. As seen from Figure 2, the Word Length effect at fixations 0 and 1 consisted of the eye fixating longer on short words than on long ones, a surprising finding already noted in O'Regan (1975). The Peripheral Content effect at fixation 0 was the result of longer fixations when peripheral vision was inappropriate.

The existence of a Peripheral Content effect on fixation duration 0 shows that information about individual letters in the critical region had indeed been collected by peripheral vision while the eye was at fixation -1 . In particular, when this information is inappropriate, the fixation in the critical region is longer, suggesting that more processing must be done when the eye arrives in the critical region.

An analysis of variance was also done on the $p r o b$ ability of making a regression at each of the saccades $-2 /-1$ through $2 / 3$. There is a significant effect of Word Length at saccade $1 / 2[F(1,20)=4.4, p<.05]$, there being fewer regressions in the case in which the critical region contains a three-letter word followed

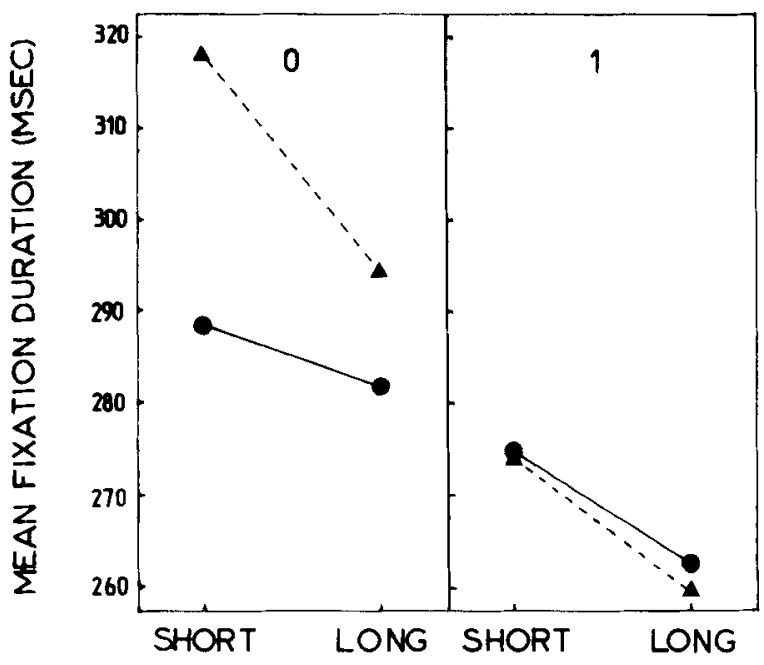

Fïgure 2. Mean fixation durations for fixations 0 and 1 . Legends as for Figure 1. 
by a medium-length word (regression probabilities for normal and inappropriate conditions $=.11$ and .14 , respectively) than in the case in which it contains a single long word (regression probabilities $=.18$ and .21). There are no other significant effects and, in particular, no effect of inappropriate peripheral content.

Up to this point, it therefore appears that fixation-0 duration is the only aspect of eye-movement behavior that reflects linguistic processing of information beyond six letters from the fixation point. This conclusion is, however, not warranted. Note first that the effect of Inappropriate Peripheral Content on saccade sizes and on regression probabilities, although statistically not significant, has always been in the direction predicted by the idea that processing is slowed down by inappropriate peripheral information at fixation -1 . The reason the effects never reach significance may be that this slowing can occur in a variety of ways over a period of several seconds and thus may not be evident when any one measure, such as fixation duration, saccade size, or regression probability, is taken individually. However, total reading time is a combined measure that might show a stronger effect of linguistic processing of information available at fixation -1 . For each of the four experimental conditions, Figure 3 shows the mean position that the eye had reached at each moment in time during the reading of the sentences. Reading proceeds at the same speed in the four conditions until the eye reaches the critical region, but the curves then separate. Reading occurs faster in the "Long" conditions, because the critical region contains one and not two

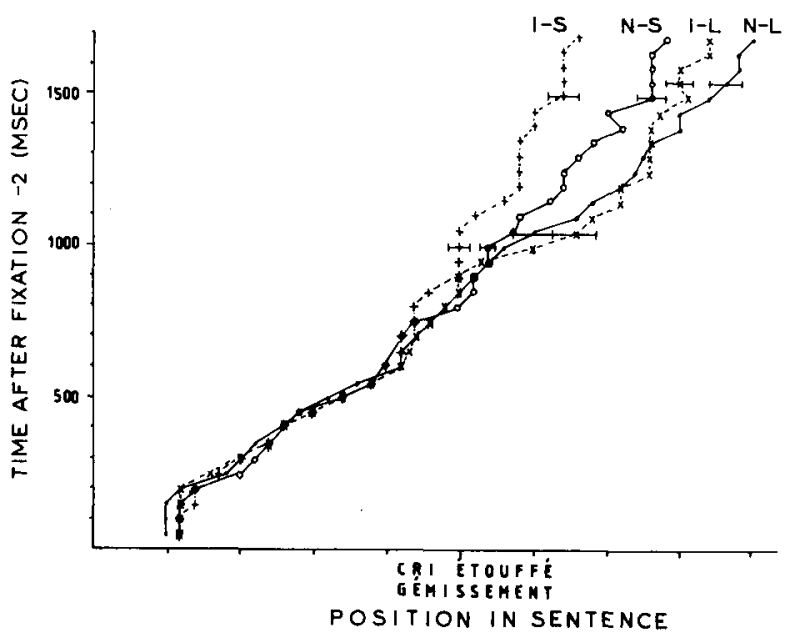

Figure 3. Mean position in the sentence that the eye has reached at each moment in time following the occurrence of fixation $\mathbf{- 2}$. Horizontal bars show examples of one standard error on each side of the mean eye position. The position occupied by the critical region in the sentence is shown by the examples CRI ÉTOUFFÉ and GÉMISSEMENT. N: NORMAL; I: INAPPROPRIATE; L: LONG; S: SHORT. words. There is an effect of Inappropriate Peripheral Content, which is particularly strong for the "Short" condition: For example, the "mean eye" reaches the last letter of the critical region at time $1,100 \mathrm{msec}$ on the abscissa for normal peripheral content, but only at time $1,500 \mathrm{msec}$ for inappropriate peripheral content. This $400-\mathrm{msec}$ difference in time taken to reach a given position is substantial compared with the 20-msec effect of inappropriate peripheral content on fixation duration 0 . This shows that the perturbation caused by the inappropriateness of peripheral content at fixation -1 is not restricted to the following fixation duration, but affects subsequent eye-movement behavior diffusely for at least $1 \mathrm{sec}$.

\section{Convenient Viewing Position}

Another aspect of the data presented earlier that requires comment is the finding that fixation duration 0 on Short Words was longer than on Long Words. The same effect was found even more strongly in O'Regan (1975) in a task in which the subject moved his eyes from an initial fixation point to a peripheral stimulus in order to decide whether it was a word or a nonword. An analysis of the present data as a function of the precise position in the critical region where the eye lands was done. Although tentative, this analysis suggested that there exist two strategies in the scanning of a long word. If the eye fixates too near the beginning or end of a long word, the fixation is foreshortened, and a second fixation is made in the word. Frequent occurrences of such foreshortened first fixations near the beginning of a long word appear to explain why fixation 0 for long words was shorter than for short words. An experiment has been done (cf. O'Regan, in press) in which, by displacing the whole sentence being read, the eye is forced to land nearer the beginning of a word than it otherwise would have. Shorter fixation durations are also found under such conditions. O'Regan (in press) suggested that these effects arise for the following reason. Combined with lexical constraints, visual information extracted from a fixation near a long word's ends is generally insuffficient to allow the word to be identified, and a second fixation must be made. However, from a fixation point at a "convenient viewing position" near the middle or left of middle of a long word, the whole word can be identified, but more time is needed. Rayner (1979) has suggested a "preferred viewing location," similar to the present "convenient viewing position," with the following difference in emphasis. Whereas Rayner's preferred viewing location is defined as the position that the eye tends to fixate, convenient viewing position further stresses the idea that if the eye does not fixate this position (e.g., because of inaccurate saccade control), then it compensates by making more fixations, of shorter durations, within the word. 


\section{CONCLUSIONS}

The following facts about eye guidance in reading have been assembled.

(1) From O'Regan (1979), it appears that within six letters of the momentary fixation point, linguistic processing (i.e., combining linguistic constraints with information from the visual field) takes place sufficiently quickly to influence the duration of the current fixation and the size of the next saccade to be made.

(2) The present experiment shows that, beyond six letters, linguistic processing still occurs, but it is slow. It does not influence the current fixation duration or the size of the next saccade made. It slightly reduces the duration of the following fixation. It does, however, substantially decrease reading time by speeding up ocular behavior over a period of about $1 \mathrm{sec}$ following the instant at which the information was gathered. This faster ocular behavior cannot be attributed to a change in one particular eye-movement parameter, but only to a combination of such changes.

(3) Easier-to-extract information about word length beyond six letters from the fixation point immediately influences saccade size, the eye making a larger saccade when approaching a long word than when approaching a short word.

(4) Fixation in long words tends to be of shorter duration than in short words. This may be because, for long words, processing is often distributed over two short-duration fixations instead of over one of longer duration.

\section{REFERENCES}

Abrams, S. G., \& Zuber, B. L. Some characteristics of information processing during reading. Reading Research Quarterly, 1972, 8, 40-51.

Bouma, H. Visual interference in the parafoveal recognition of initial and final letters of words. Vision Research, 1973, 13, 767-782.

Gillitand, A. $R$. The effect on reading of changes in the size of type. Elementary School Journal, 1923, 24, 138-146.

HABER, R. N. Control of eye movements during reading. In R. A. Monty \& J. W. Senders (Eds.), Eye movements and psychological processes. Hillsdale, N.J: Erlbaum, 1976.

HochBerG, J. Components of literacy: Speculations and exploration research. In $\mathrm{H}$. Levin \& J. P. Williams (Eds.), Basic studies on reading. New York: Basic Books, 1970.
Huky, E. B. On the psychology and physiology of reading. I. American Journal of Psychology, 1900, 11, 283-302.

Javal, K. E. Essai sur la physiologie de la lecture. Annales d'Oculistique, 1879, 82, 243-253.

MoConkie, G. W., \& Rayner, K. The span of the effective stimulus during a fixation in reading. Perception \& Psychophysics, 1975, 17, 578-586.

O'REgan, J. K. Structural and contextual constraints on eye movements in reading. Unpublished doctoral dissertation, University of Cambridge, 1975.

O'REGAN, J. K. A better horizontal eye movement calibration method: Subject controlled smooth pursuit and zero drift. Behavior Research Methods \& Instrumentation, 1978, 10, 393-397.

O'REgAN, J. K. Saccade size control in reading: Evidence for the linguistic control hypothesis. Perception \& Psychophysics, 1979, 25, 501-509.

O'REgAN, J. K. Convenient viewing position. In D. Fisher, J. W. Senders, \& R. A. Monty (Eds.), Eye movements: Cognition and visual perception. Hillsdale, N.J.: Erlbaum, in press.

RAYNER, K. The perceptual span and peripheral cues in reading. Cognitive Psychology, 1975, 7, 65-81.

RAYNER, K. Eye guidance in reading: Fixation locations within words Perception, 1979, 8, 21-30.

RaYner, K., \& MoConkiE, G. W. What guides a reader's eye movements? Vision Research, 1976, 16, 829-837.

\section{NOTE}

1. I will use "noncentral vision" or "peripheral vision" to designate all parts of the visual field outside "central vision." I define "central vision" as that part of the visual field extending about three letters on each side of the fixation point (independently of viewing distance) in which letters in a random string of letters can be accurately identified. Other authors have used the terms "foveal" and "parafoveal," but I prefer "central" and "peripheral" for two reasons. First, physiologically, there is no sudden drop in retinal cell density as we move outward from the center of the eye. Second, functionally, acuity measurements show no sudden change. On the contrary, the acuity of the eye decreases immediately starting from the eye's center. Up to a field size of $20 \mathrm{deg}$, the decrease goes as the reciprocal of the distance from the center: Doubling the distance halves the acuity. An important consequence of this is that, for a given typeface, changing viewing distance does not change the number of letters that can be seen clearly: If viewing distance is doubled, acuity must be doubled to see the same kind of letter. The size of the effective visual field is therefore halved, giving back the same number of identifiable letters. The validity of this argument can be confirmed by holding a string of random letters at different distances. It will be found that, for a large range of viewing distances, about three letters on each side of the fixation point can be identified.

(Received for publication July 26, 1979; revision accepted March 10, 1980.) 